\title{
Report of a case of cerebral aneurysm in an adult developing apparently de novo
}

\author{
CARL J. GRAF AND W. B. HAMBY \\ From Buffalo General Hospital, Buffalo 3, N.Y., and the Cleveland Clinic Foundation
}

The aetiology and pathogenesis of cerebral arterial aneurysms, particularly of the berry type, have been the subject for much study and speculation. The bulk of this work up to 1952 was reviewed and summarized in Hamby's (1952) monograph on intracranial aneurysms and will not be reiterated here. No more specific information seems to have developed subsequently. A fair summary of majority opinion on the subject appears to be that these lesions develop on the basis of some congenital peculiarity in the vessel walls, either of Forbus (1930) or Bremer (1943) type, influenced by variable, vaguely understood, post-natal circulatory changes.

The importance of there being several of these aneurysms present has become recognized and most surgeons now consider it important to perform bilateral angiograms in studying a case of subarachnoid haemorrhage. A number of instances have been reported of patients having subsequent bouts of haemorrhage from aneurysms contralateral to the one treated originally. Earlier, the contralateral vessels might not have been visualized and it was presumed that the second aneurysm was present, but silent, at the time of the first investigation. If the original aneurysm was treated by proximal carotid ligation, alterations in contralateral blood flow might conceivably have set the stage for later haemorrhage from the second one.

As far as we have been able to ascertain, no instance has been reported of an aneurysm developing de novo and bleeding after satisfactory, negative angiography. Such a case is the basis for this report.

\section{CASE REPORT}

FIRST ADMISSION L.G., a 39-year-old woman, was admitted to the Buffalo General Hospital on 19 February 1960. In September 1959, while dancing, she slipped and fell, striking the back of her head. She did not lose consciousness. Intermittently thereafter she complained of occipital headaches. On 15 February 1963, while taking aspirin for her usual headache, she noticed blurred vision, felt weak and had sharp, severe, occipital headache and then 'passed out'. She was discovered unconscious and then, after being taken to a local hospital, awoke orientated and vomited. She complained of occipital headache which persisted until her admission to Buffalo General Hospital four days later.

Examination showed a pale, alert, fully-orientated woman with blood pressure of $178 / 94 \mathrm{~mm}$. $\mathrm{Hg}$. There was early optic disc elevation of 1-2 diopters. Lumbar puncture revealed grossly bloody cerebrospinal fluid under $450 \mathrm{~mm}$. of pressure. On 23 February, bilateral carotid and left vertebral angiography was accomplished, showing a $3 \mathrm{~mm}$. aneurysm on the right middle cerebral artery at the level of the trifurcation (Fig. 1), associated with marked constriction of all the mid-cerebral branches and of the right anterior cerebral artery. The left carotid and left vertebral systems were clear (Fig. 2). On the evening of the angiogram, left hemiparesis was noted, greater in the arm than in the leg. Speech was slurred and the patient was incontinent. A recurrent haemorrhage and perhaps an intracerebral clot were suspected; lumbar puncture was repeated and xanthochromic cerebrospinal fluid was found to be under $490 \mathrm{~mm}$. of pressure. On 24 February the patient still complained of intense headache and her neck was stiff. No spontaneous left extremity movement was evident, though gross movement occurred on painful stimulation. Papilloedema increased and Babinski's sign was positive on the left side.

On 25 February, through a right frontal craniotomy, the aneurysm was exposed, clipped from the parent trunk, and the major portion of it excised. That evening the left hemiparesis was more pronounced and the patient was quite drowsy. On 3 March she responded well, was emotionally labile, and had a left hemiparesis. On 7 March the patient was alert and followed commands, but showed no spontaneity. Two days later she was confused, irrational, and paid little attention to request or suggestion. Although she said she was able to 'feel' her left hand, she seemed to ignore it. On 10 March repeat carotid angiography showed good middle cerebral vessels and absence of the aneurysm. The patient was discharged on 11 March with a spastic hemiparesis, somewhat improved.

SECOND ADMISSION Mrs. L. G. was re-admitted to the hospital on 1 March 1963. It was said that since her previous admission, the hemiparesis had improved and that she had carried out the duties of a housewife. About six months following the operation, she began to have episodes of 'stiffening' of the left leg, associated with loss 


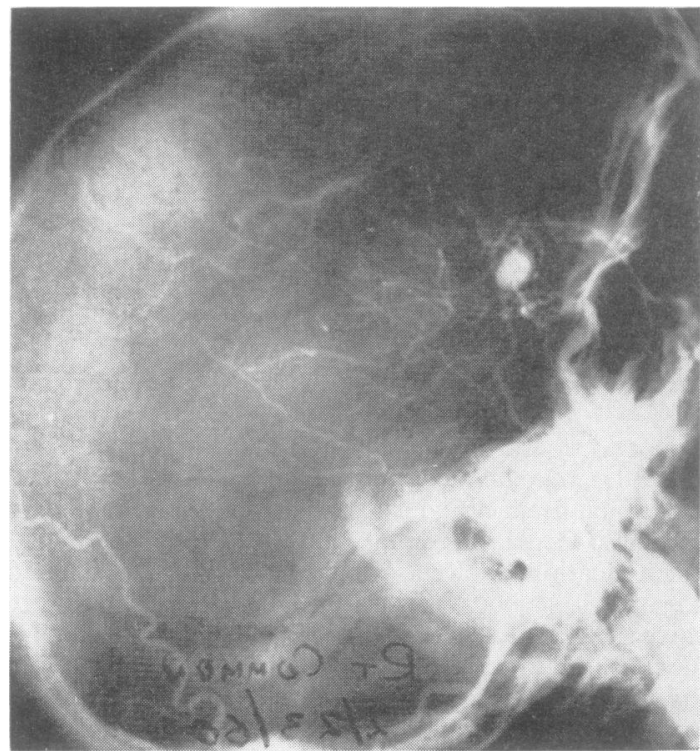

FIG. 1A

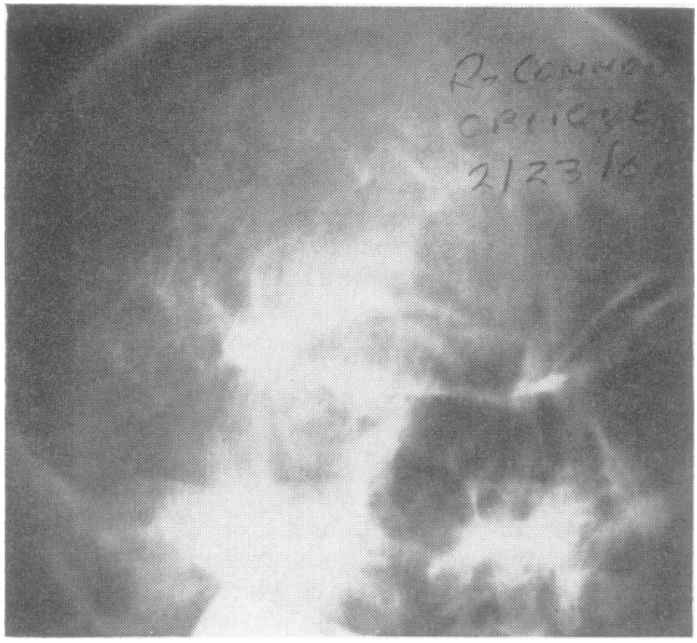

FIG. 1B

FIG. 1A and B. Arteriogram, right common carotid injection, on 23 February 1960.

of awareness, On the day of admission, such an attack occurred, followed by nausea, vomiting, and fainting. Upon admission to a local hospital, she was described as being semi-stuporous and remained so until her transfer to the Buffalo General Hospital. There was a question of her having had a generalized convulsive seizure just before admission.

Examination showed a mentally obtunded woman with temperature of $102^{\circ} \mathrm{F}$. She would open her eyes and look at the examiner, but she resisted ophthalmoscopic examination. She turned herself in bed. She did not

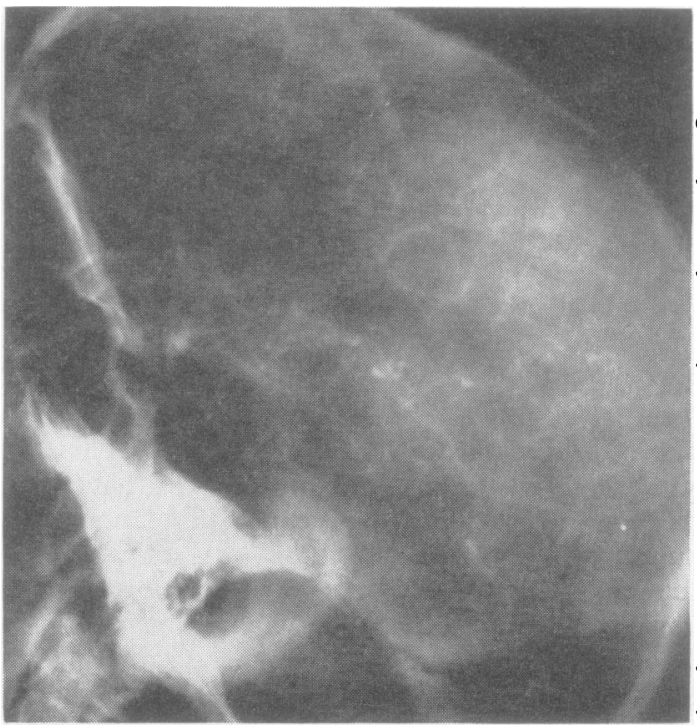

FIG. $2 \mathrm{~A}$

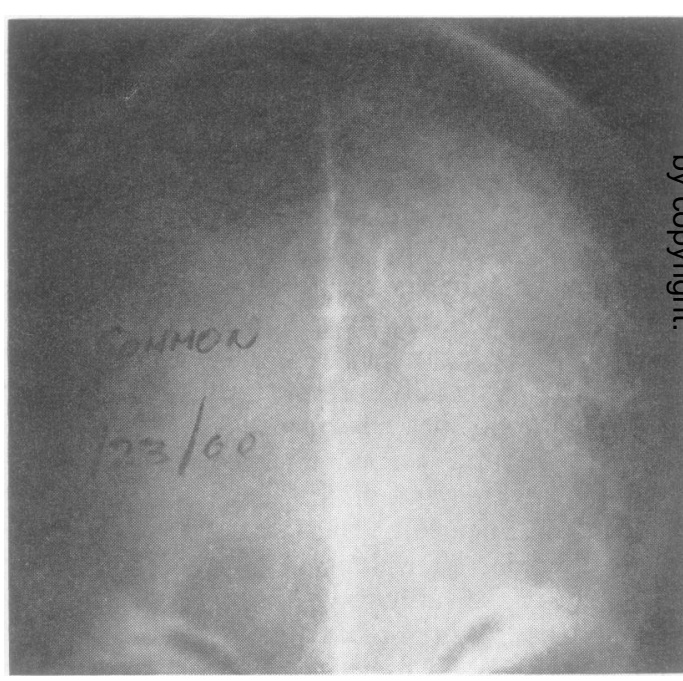

FIG. 2B

FIG. 2A and B. Arteriogram, left common carotid injection, on 23 February 1960.

follow any command. The neck was rigid; she moved all extremities, including a spastic left arm and leg.

The cranial nerves were undisturbed. The left deep $\square$ tendon reflexes were more active than the right, with bilateral Babinski's sign. There was questionable diminution of pain sensation in the left leg.

Lumbar puncture revealed bloody cerebrospinal fluid. $N$ On the next day the patient was perhaps less aware. The $\mathrm{N}$ right arm and hand moved spontaneously. The patient $\omega$ would raise this extremity, look at it inquisitively, $\widetilde{\sigma}$ turning it in what seemed to be purposeful activity. Theo 


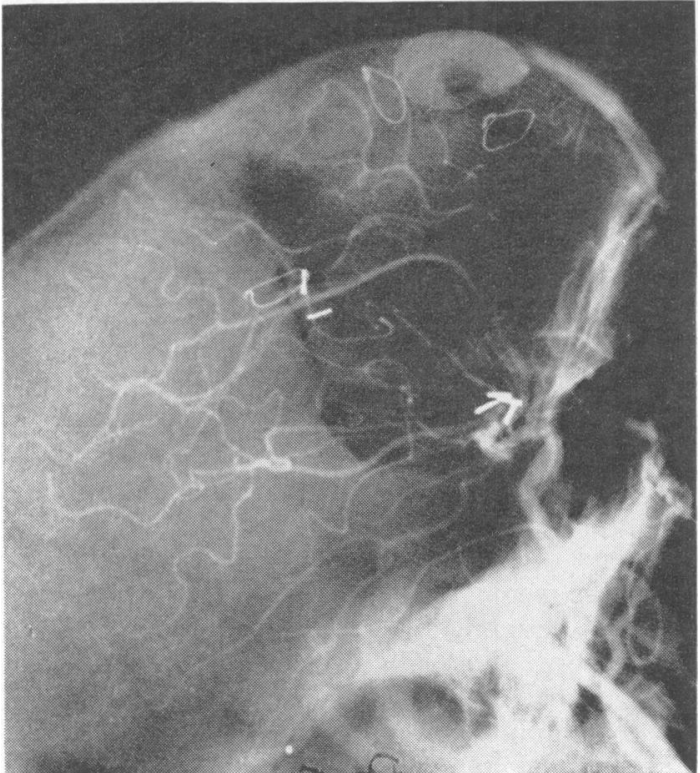

FIG. $3 A$

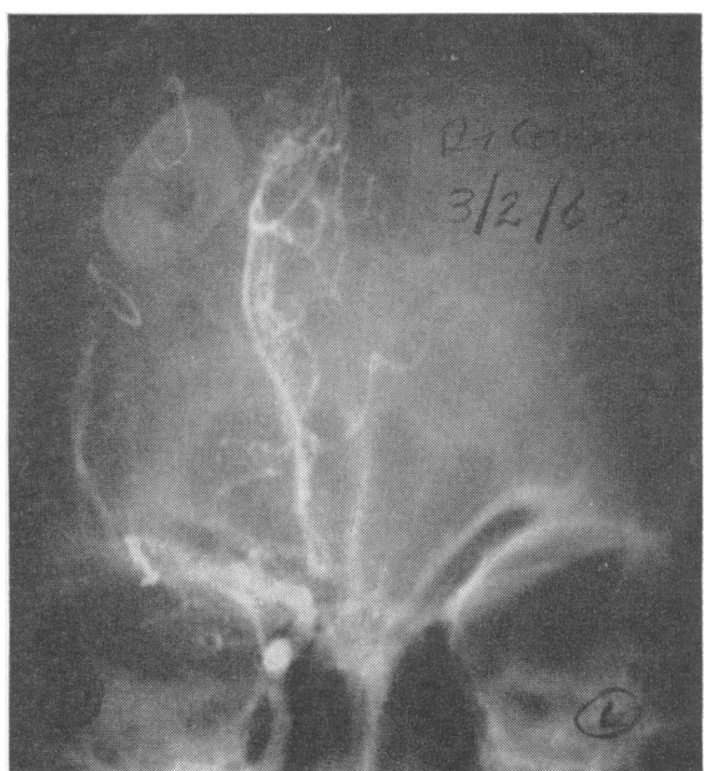

FIG. 3B

FIG. 3A and B. Arteriogram, right common carotid injection, on 2 March 1963. No evidence of right middle cerebral aneurysm, its site indicated by clips.

left arm was held flexed at the elbow with slight pronation of the forearm and flexion-pronation at the wrist. There was little spontaneous right finger movement, but the left arm responded to vigorously painful stimulation. The left paresis seemed worse than on the day before, though we were unable to evaluate it from the preadmission state. The Babinski signs had disappeared.
This patient had had a recurrent subarachnoid haemorrhage, of undetermined origin. It was considered likely that this haemorrhage was from a new aneurysm or from one previously unrecognized, associated with a right intracerebral haematoma.

On 2 March bilateral carotid angiograms were made. On the right side (Fig. 3), that of the previous operation,

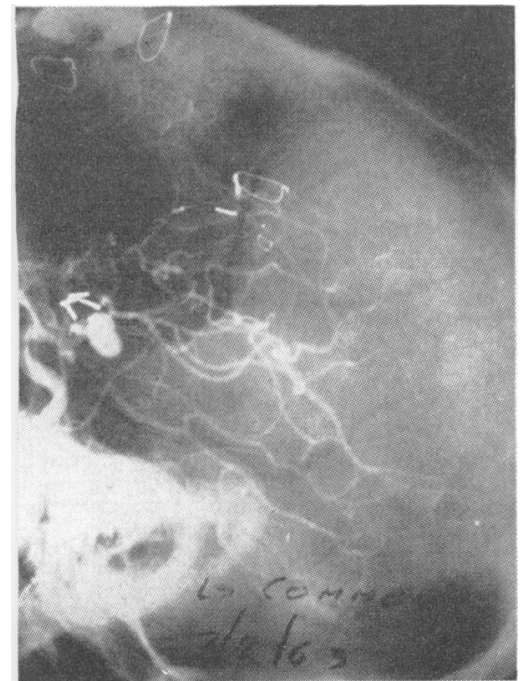

4A

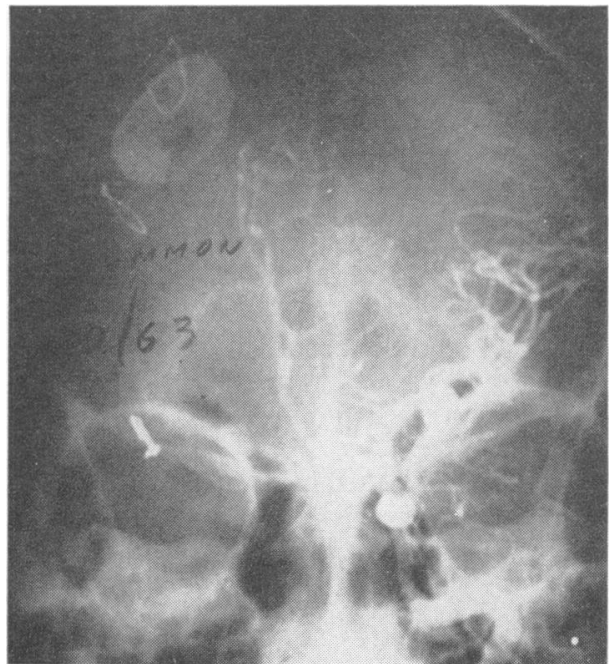

FIG. 4B

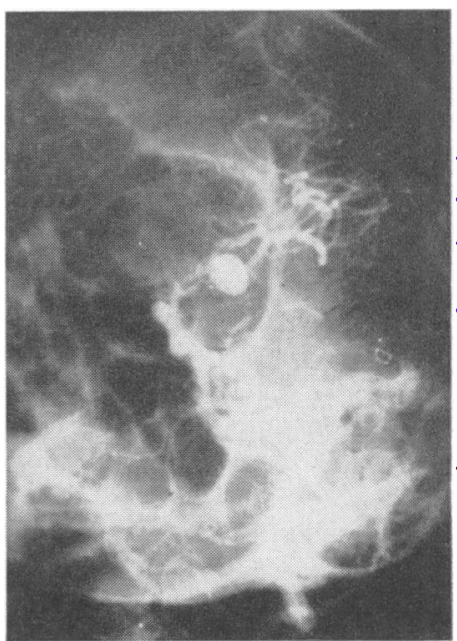

FIG. 4C

FIG. 4A, B, and C. Arteriogram, left common carotid injection, on 2 March 1963. 
the carotid tree was well demonstrated. There was no evidence of aneurysm. Several metallic clips were seen at the site of the previously demonstrated aneurysm, now successfully occluded. The anterior cerebral arteries were shifted sharply to the right. On the left side (Fig. 4, A, B, C) a saccular aneurysm, larger than that demonstrated three years before on the right side, was seen arising from the bifurcation of the left middle cerebral artery. The anterior cerebral artery was shifted to the right. The middle cerebral artery was displaced upward and medially in the temporal fossa and its peripheral branches were separated from the skull in the parietal area. The appearance was that of a left middle cerebral artery aneurysm, with associated intracerebral and Sylvian fissure clot and subdural haematoma.

The patient was operated upon immediately through a left fronto-temporal craniotomy. Haematoma was evacuated from the subdural space, the Sylvian fissure, and the left temporal lobe. The neck of the aneurysm was occluded with clips.

On the first post-operative day, the patient's attitude suggested the decerebrate state. She was treated thereafter with hypothermia, nasogastric feeding, and antibiotics. On the following day stimulation exaggerated the decerebrate posture. Babinski's sign was present bilaterally. Electrolyte balance was well maintained. On 5 March the patient vomited and aspirated, developing right pulmonary atelectasis, and the mediastinum shifted.

Lumbar puncture on 6 March revealed pink cerebrospinal fluid under $250 \mathrm{~mm}$. of pressure. The patient's condition deteriorated and she expired on 7 March. Post-mortem examination was not permitted.

\section{DISCUSSION}

A case is presented of a patient having subarachnoid haemorrhage from a right middle cerebral aneurysm. Bilateral angiograms revealed no evidence of a lesion on the left side. The aneurysm was occluded without interrupting the middle cerebral circulation. Post-operative angiograms confirmed these comments.

The patient lived for three years with evidence of moderately severe right cerebral damage. She then developed a fresh subarachnoid, left subdural, and left temporal intracerebral haemorrhage. Bilateral carotid angiography showed the original right cerebral vessels to be intact, and a new aneurysm, larger than the original contralateral one, on the left middle cerebral artery.

The therapeutic implications here are disturbing. We have always felt that if a single aneurysm were demonstrated by adequate bilateral carotid angio- graphy and obliterated, the patient could be given $\mathbb{D}$ fairly positive assurance that he would not have further difficulty. If, however, aneurysms can arise de novo, it would seem that there is less security in assuring such a patient that the successful treatment of one aneurysm solves his aneurysm problem.

The cause of the second aneurysm in this case is $\frac{\overline{\mathrm{N}}}{\mathrm{g}}$. obscure. Pre- and post-operative angiograms at the first episode showed no gross contralateral one. It is:unlikely that an aneurysm as large as this was present at the time of the first haemorrhage ando escaped visualization on angiograms made two음 weeks apart.

According to Bremer's concept (1943), micro- $\mathbb{Q}$ scopic vessels may persist as remnants of the embryonic capillary net. These are not necessarily ${ }_{-}^{\text {s }}$ blind end arteries; they may be vasa vasorum or. supply the meninges. Bremer was under the impres- $\overrightarrow{\vec{\omega}}$ sion that these might arise in an arterial wall, and persist there, potentially open. If inflow into them expanded the lumen, an aneurysm could develop. The lesion in our case may have been of this nature; $i$ or perhaps the lesion was very small and obscured on iv the angiograms by surrounding vessels, to enlarge $\vec{G}$ later as suggested. Haemodynamics should not have $\omega$

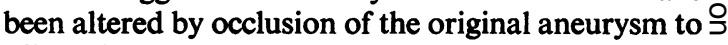
affect significantly the site of the second, as mig $-\overrightarrow{-}$ have been postulated had the original lesion begn $\frac{D}{0}$ treated by proximal ligation. It is more reasonable to. assume that a structural defect of some type w\$ु $\vec{\varphi}$ primarily the 'anlage' for aneurysmal development, $\rightarrow$ the physical characters of blood flow and blood pressure being secondary factors in the development of this lesion.

\section{SUMMARY}

A case is presented of a second, contralateral $\overline{\bar{O}}$ aneurysm developing in an adult on a middle cerebral artery previously normal on angiograms. Delusions of security after cure of a single demonstrable aneurysm must be abandoned. Possible mechanisms of the development of such lesions are? discussed.

\section{REFERENCES}

Bremer, J. L. (1943). Congenital aneurysms of the cerebral arteries. 음 An embryologic study. Arch. Path., 35, 819-831.

Forbus, W.D. (1930). On the origin of miliary aneurysms of superficial $D$ cerebral arteries. Bull. Johns Hopk. Hosp., 47, 239-284.

Hamby, W. B. (1952). Intracranial Aneurysms, pp. 109-132. Thomas, Springfield, Illinois. 